\title{
Death by ubiquitylation
}

\section{$(4$}

...p62

mediates the aggregation of ubiquitylated caspase $8 \ldots$
The extrinsic pathway of apoptosis is initiated when certain tumour necrosis factor superfamily ligands, such as APO2L (also known as TNFSF10 or TRAIL), activate cell surface death receptors. The ligand-receptor complex interacts with FAS-associated death domain protein, an adaptor protein that recruits caspase 8 - the caspase that initiates apoptosis in this pathway - to form a death-inducing signalling complex (DISC). Caspase 8 initially forms a dimer, but it must undergo multimerization (aggregation) to be fully activated. However, the exact mechanism of caspase 8 aggregation has remained unknown. Ashkenazi and colleagues now report that, to become fully active and able to commit cells to death, caspase 8 must undergo polyubiquitylation

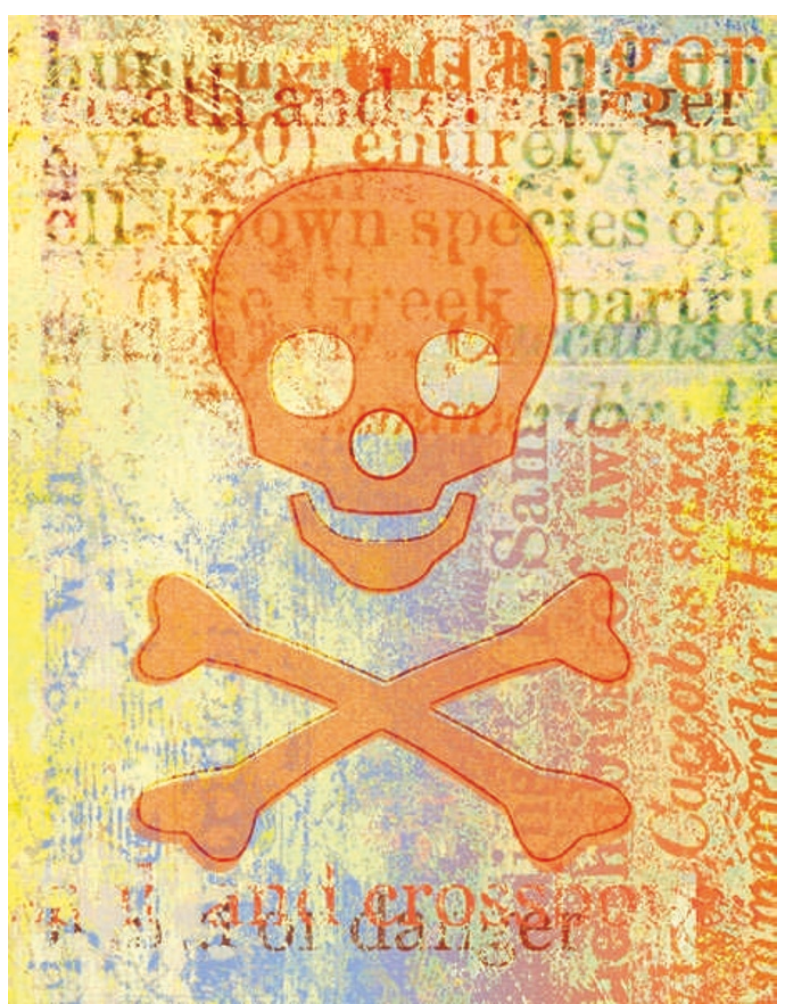

mediated by the cullin 3 (CUL3)based E3 ubiquitin ligase, followed by ubiquitin-binding protein p62 (also known as sequestosome 1)-dependent aggregation.

The authors first observed that caspase 8 is ubiquitylated in response to $\mathrm{APO} 2 \mathrm{~L}$ and that this promotes caspase 8 aggregation rather than protein degradation. Tandem mass spectrometry of the DISC isolated from ligand-treated cells revealed the presence of the E3 subunit CUL3, the deubiquitylating enzyme A20 and p62. So, why do these proteins associate with the ligand-induced DISC and how do they influence caspase 8 activity and apoptosis?

Depletion of CUL3, or its associated subunit RING-box protein 1 (RBX1), markedly decreases the amount of ubiquitylated caspase 8 in the DISC and also decreases caspase 8 activity and apoptosis. Using ubiquitin mutants and mass spectrometry, the authors determined that caspase 8 undergoes CUL3-dependent polyubiquitylation in its C-terminal region and that this can promote caspase 8 aggregation and activation even in the absence of a ligand-induced DISC. A20 reverses the CUL3-mediated ubiquitylation of caspase 8 and the associated increase in caspase activity, which suggests that A20 is present in the DISC to serve as the deubiquitylating enzyme for caspase 8 .

Finally, why is p62 associated with the DISC in cells treated with APO2L? The interaction of p62 with the DISC is dependent on CUL3-mediated ubiquitylation of caspase 8. Depletion of p62 reverses the aggregation of caspase 8 that occurs when caspase 8 is co-expressed with CUL3, and decreases caspase 8 activation. Furthermore, treatment of cells with APO2L results in the colocalization of caspase 8 and p62 to ubiquitin-rich foci in a p62-dependent manner. Together, these data suggest that p62 mediates the aggregation of ubiquitylated caspase 8 to promote its full activation and efficient apoptosis.

Thus, polyubiquitylation directly and positively influences caspase 8 in response to apoptotic stimuli and is a critical regulator of the extrinsic apoptotic pathway.

Katharine H. Wrighton

ORIGINAL RESEARCH PAPER Jin, Z. et al.

Cullin3-based polyubiquitination and p62dependent aggregation of caspase-8 mediate extrinsic apoptosis signaling. Cell 137, 721-735 (2009)

FURTHER READING Taylor, R. C. et al.

Apoptosis: controlled demolition at the cellular level. Nature Rev. Mol. Cell Biol. 9, 231-241 (2008) 